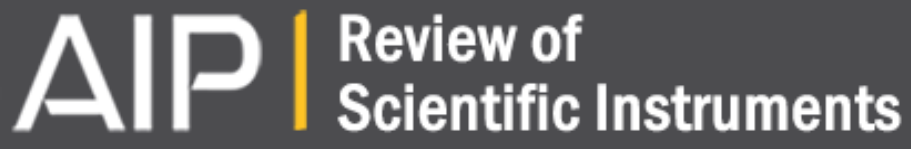

A new visible spectroscopy diagnostic for the JET ITER-like wall main chambera)

C. F. Maggi, S. Brezinsek, M. F. Stamp, S. Griph, P. Heesterman, C. Hogben, A. Horton, A. Meigs, C. Morlock, W. Studholme, K.-D. Zastrow, and JET-EFDA Contributors

Citation: Review of Scientific Instruments 83, 10D517 (2012); doi: 10.1063/1.4733734

View online: http://dx.doi.org/10.1063/1.4733734

View Table of Contents: http://scitation.aip.org/content/aip/journal/rsi/83/10?ver=pdfcov

Published by the AIP Publishing

\section{Articles you may be interested in}

Ion cyclotron resonance frequency heating in JET during initial operations with the ITER-like walla)

Phys. Plasmas 21, 061510 (2014); 10.1063/1.4884354

Enhancements to the JET poloidally scanning vacuum ultraviolet/visible spectrometersa)

Rev. Sci. Instrum. 83, 10D536 (2012); 10.1063/1.4745213

A protection system for the JET ITER-like wall based on imaging diagnosticsa)

Rev. Sci. Instrum. 83, 10D727 (2012); 10.1063/1.4738742

New diagnostic techniques and technologies at JET (invited)

Rev. Sci. Instrum. 77, 10F529 (2006); 10.1063/1.2336447

Atomic modeling and instrumentation for measurement and analysis of emission in preparation for the ITER-like wall in JET

Rev. Sci. Instrum. 77, 10F520 (2006); 10.1063/1.2236278

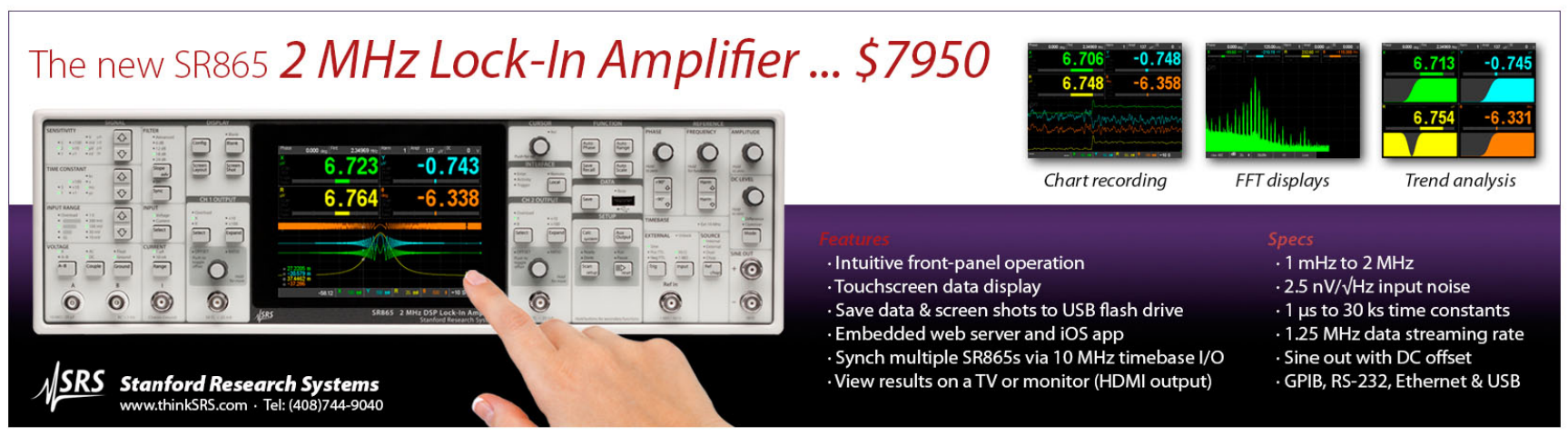




\title{
A new visible spectroscopy diagnostic for the JET ITER-like wall main chambera)
}

\author{
C. F. Maggi, ${ }^{1, b)}$ S. Brezinsek, ${ }^{2}$ M. F. Stamp, ${ }^{3}$ S. Griph, ${ }^{3}$ P. Heesterman, ${ }^{3}$ \\ C. Hogben, ${ }^{3}$ A. Horton, ${ }^{3}$ A. Meigs,${ }^{3}$ C. Morlock, ${ }^{4}$ W. Studholme, ${ }^{3}$ K.-D. Zastrow, ${ }^{3}$ \\ and JET-EFDA Contributors ${ }^{5, c)}$ \\ ${ }^{1}$ Max-Planck-Institut für Plasmaphysik, EURATOM Association, D-85748 Garching, Germany \\ ${ }^{2}$ Forschungszentrum Jülich GmbH, EURATOM Association, D-52425 Jülich, Germany \\ ${ }^{3}$ EURATOM/CCFE, Fusion Association, Culham Science Centre, Abingdon, OX14 3DB, United Kingdom \\ ${ }^{4}$ EFDA-CSU Garching, Boltzmannstrasse 2, 85748 Garching, Germany \\ ${ }^{5} J E T-E F D A$, Culham Science Centre, Abingdon, OX14 3DB, United Kingdom
}

(Presented 7 May 2012; received 4 May 2012; accepted 18 June 2012;

published online 17 July 2012)

In preparation for ITER, JET has been upgraded with a new ITER-like wall (ILW), whereby the main plasma facing components, previously of carbon, have been replaced by mainly $\mathrm{Be}$ in the main chamber and $\mathrm{W}$ in the divertor. As part of the many diagnostic enhancements, a new, survey, visible spectroscopy diagnostic has been installed for the characterization of the ILW. An array of eight lines-of-sight (LOS) view radially one of the two JET neutral beam shine through areas (W coated carbon fibre composite tiles) at the inner wall. In addition, one vertical LOS views the solid W tile at the outer divertor. The light emitted from the plasma is coupled to a series of compact overview spectrometers, with overall wavelength range of 380-960 nm and to one high resolution Echelle overview spectrometer covering the wavelength range $365-720 \mathrm{~nm}$. The new survey diagnostic has been absolutely calibrated in situ by means of a radiometric light source placed inside the JET vessel in front of the whole optical path and operated by remote handling. The diagnostic is operated in every JET discharge, routinely monitoring photon fluxes from intrinsic and extrinsic impurities (e.g., Be, $\mathrm{C}, \mathrm{W}, \mathrm{N}$, and $\mathrm{Ne}$ ), molecules (e.g., $\mathrm{BeD}, \mathrm{D}_{2}, \mathrm{ND}$ ) and main chamber and divertor recycling (typically $\mathrm{D} \alpha, \mathrm{D} \beta$, and $\mathrm{D} \gamma$ ). The paper presents a technical description of the diagnostic and first measurements during JET discharges. [http://dx.doi.org/10.1063/1.4733734]

\section{INTRODUCTION}

In preparation for ITER, JET has been upgraded with a new ITER-like wall ${ }^{1,2}$ (ILW), whereby the main plasma facing components, previously of carbon fibre composite (CFC), have been replaced by mainly Be in the main chamber and $\mathrm{W}$ in the divertor. An extensive upgrade of spectroscopic diagnostics has accompanied the installation of the ILW in order to allow for its characterization in terms of impurity source distributions, erosion rates and migration patterns. As part of the many diagnostic enhancements, a new, survey, visible spectroscopy diagnostic has been installed to monitor one of the 2 JET neutral beam (NB) shine through areas in the main chamber. These are areas of recessed $\mathrm{W}$ coated CFC tiles, comprising a portion of the inner wall guard limiter (IWGL) and the adjacent inner wall tiles. They are the only $\mathrm{W}$ main chamber plasma facing components of the new JET main chamber, the remainder being made of Beryllium. An additional, vertical line-of-sight views the solid $\mathrm{W}$ tile at the outer divertor. The new diagnostic was installed on JET in 2010, commissioned and calibrated in 2011 and became

\footnotetext{
a) Contributed paper, published as part of the Proceedings of the 19th Topical Conference on High-Temperature Plasma Diagnostics, Monterey, California, May 2012.

b) Author to whom correspondence should be addressed. Electronic mail: Costanza.Maggi@ccfe.ac.uk.

${ }^{c}$ See the Appendix of Romanelli et al., Proceedings of the 23rd IAEA Fusion Energy Conference 2010, Daejeon, Korea.
}

operational from the first day of the JET experimental campaigns in August 2011. This paper describes the setup of the new survey spectrometer systems (known as KS8 in JET) and gives first experimental results.

\section{VIEWING GEOMETRY}

An array of eight radial lines-of-sight (LOS) to the inner wall view the JET neutral beam shine through area at octant 7 of the tokamak just above the midplane. These tiles are recessed with respect to the other main plasma facing components. The 8 LOS are poloidally and toroidally distributed, with 3 viewing the IWGL and 5 the adjacent inner wall tiles, as shown in the layout of Figure 1. The LOS spot diameter is $5.2 \mathrm{~cm}$ at the inner wall. An additional LOS views the solid W tile at the outer divertor (tile 5) from a vertical port through a double sapphire window (primarily installed for IR diagnostics) and has a spot diameter of $23 \mathrm{~cm}$ at the divertor target. A poloidal cross section of the KS8 viewing geometry is shown in Figure 2. The final diagnostic alignment was carried out at the JET wall operating temperature of $200{ }^{\circ} \mathrm{C}$. For the radial LOS the optical head consists of a quartz lens assembly and a fibre holder housing the fibre optics, installed on a bracket bolted in front of a Si-Si window. The plasma light is transferred by $600 \mu \mathrm{m}$ quartz fibers about $70 \mathrm{~m}$ away from the tokamak to a spectrometer room (low radiation and stable temperature area) where the spectrometers are located. 


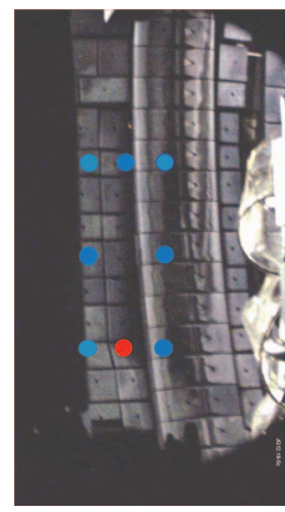

FIG. 1. Layout of the 8 radial LOS of the KS8 diagnostic to the JET inner wall neutral beam shine through area (W coated CFC tiles) at octant 7: 3 LOS viewing the inner wall guard limiter and 5 the inner wall (blue circles represent views coupled to COS, red circle represents a view coupled to EOS).

\section{SPECTROMETERS AND CCD DETECTORS}

In the spectrometer room the plasma light is coupled to 32 compact overview spectrometers (COS) and one high spectral resolution Echelle overview spectrometer (EOS). The COS are mini Czerny-Turner spectrometers with fixed grating and medium spectral resolution, $75 \mathrm{~mm}$ AvaBench (model AvaSpec-ULS2048L-USB2-RM with USB2/RS-232 interface, from Avantes). The output spectra from the COS systems are imaged onto 2048 pixel CCD detectors (frame transfer). Twenty four COS are equipped with $2400 \mathrm{l} / \mathrm{mm}$ grating and cover the wavelength range 380-635 nm (8 COS for the range $380-471 \mathrm{~nm}, 8$ for the range $467-541 \mathrm{~nm}$, and 8 for the range $587-635 \mathrm{~nm}$ ), while $8 \mathrm{COS}$ cover the 620-960 $\mathrm{nm}$ range with $830 \mathrm{l} / \mathrm{mm}$ gratings. Four COS spectrometers are mapped to each LOS, thus providing an overall wavelength coverage of 380-960 nm per LOS. This configuration is obtained by splitting the incoming plasma light from a $600 \mu \mathrm{m}$ fiber into 4 bundles of $100 \mu \mathrm{m}$ fibers optimized to the spectrometers entrance slit, as shown schematically in Figure 3. In particular, at the spectrometers patch panel the light from the $600 \mu \mathrm{m}$ fiber is divided into $21 \times 90 \mu \mathrm{m}$ fibers with thin outer cladding (outer diameter: $107 \mu \mathrm{m}$ ), which are then redistributed into 4 bundles of fibers, with, respectively, $6,6,5$, and 4 fibers each in a row. The light from the fiber bundle is imaged onto the spectrometer's slit with, in vertical

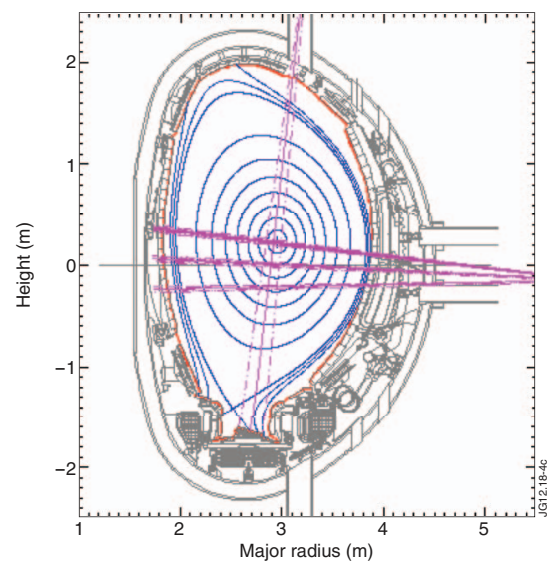

FIG. 2. Poloidal cross section of the KS8 viewing geometry.

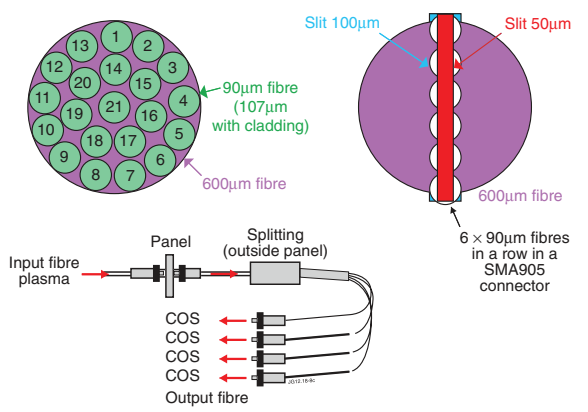

FIG. 3. Schematic of the fibre coupling arrangement utilized for the COS spectrometers at the patch panel: the light from the input $600 \mu \mathrm{m}$ fibre (from the plasma) is divided into $21 \times 100 \mu \mathrm{m}^{2}$ output fibres, which are then redistributed into 4 bundles of 6, 6, 5, and 4 fibres stacked in rows. The configuration with a row of 6 fibres is shown on the right. Each bundle constitutes an output fibre optimized to a COS entrance slit (4 COS coupled to each LOS).

direction, the rows of 6 fibers for the lower response devices (shorter wavelength) and the rows of 4 fibers for the more efficient, near-infrared systems. Some light losses cannot be avoided due to the spectrometers slit widths being narrower than $100 \mu \mathrm{m}$ for most of the COS (as described below), however the resulting filling factor for the whole system of spectrometers is $\sim 1 / 3$. For 6 LOS the corresponding COS are equipped with $50 \mu \mathrm{m}$ slits, providing medium spectral resolution. One LOS is coupled to a $100 \mu \mathrm{m}$ slit width system for faster time acquisition, while the divertor LOS is coupled to a higher spectral resolution system, with $25 \mathrm{~mm}$ slit width. For each JET discharge, each COS is individually configurable with respect to triggering, exposure time and number of spectra acquired. The COS are operated at a typical exposure time of $100 \mathrm{~ms}$ for survey (minimum exposure time is $2 \mathrm{~ms}$ ). The data acquisition is automatic, in external sync during each JET discharge. One of the KS8 radial LOS is connected to an Echelle overview spectrometer (Spectrelle 20000p, Systematix and GWU Lasertechnik GmbH cooperation, $110 \mathrm{~mm}$ focal length and direct fibre input with SMA905 connector). The system provides the simultaneous wavelength range of 365-720 $\mathrm{nm}$ in cross dispersion, ${ }^{3}$ through the combined use of two dispersive elements: a highly dispersive Echelle grating (ruling: $31.6 \mathrm{~g} / \mathrm{mm}$, blaze angle: $76^{\circ}$ ) for wavelength dispersion in the horizontal direction and a highly dispersive prism as order sorter, separating the orders in the vertical direction. This is combined with high spectral

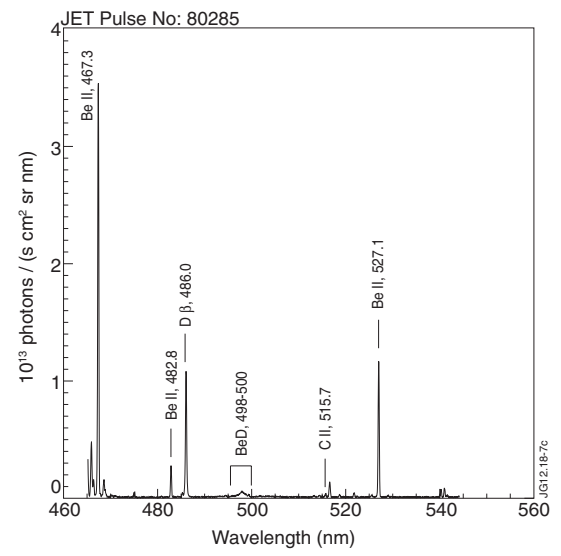

FIG. 4. Example of a COS spectrum (inner wall view) from a JET discharge. 


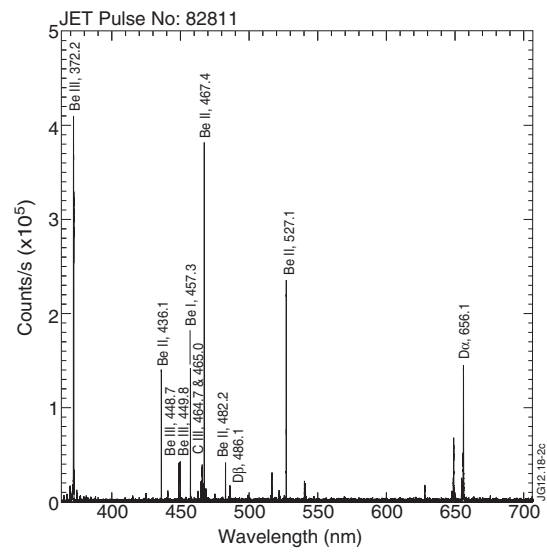

FIG. 5. Example of EOS spectrum from the limiter phase of JET discharge \#82811 (inner wall view).

resolution (resolving power $\lambda / \Delta \lambda \sim 20,000$ ) and high throughput (F-number of 3.6) over $\sim 45,000$ spectral channels. The light is imaged onto a back-illuminated ANDOR iXon ${ }^{\mathrm{EM}+} \mathrm{CCD}, 1024 \times 1024,13 \mu \mathrm{m}^{2}$ pixel size (model \#DU$888 \mathrm{E}-\mathrm{C} 00-\mathrm{ABV}$ ). The system is typically operated at the maximum time resolution of $120 \mathrm{~ms}$ (at $10 \mathrm{MHz}, 14 \mathrm{bit}$ ). A mechanical shutter (Uniblitz LS6T2) has been installed and coupled to the externally triggered frame transfer CCD operation in order to correct for the image smearing induced by the vertical frame transfer of the CCD. The data acquisition is automatic, in external sync during each JET discharge, with both the full 2D images and the reconstructed spectra vs wavelength being recorded (typical JET pulse duration is $30 \mathrm{~s}$ ). The EOS system and its applications will be described in greater detail in a separate paper. The data collected by the COS and EOS systems are then transferred to the memory of personal computers (PCs) using dedicated data acquisition software written for the JET computer system. The PCs are controlled automatically by HTTP commands from a central JET UNIX data acquisition computer over ethernet connections. Data from each JET discharge are acquired on the PCs and are then automatically archived on the main JET data storage system.

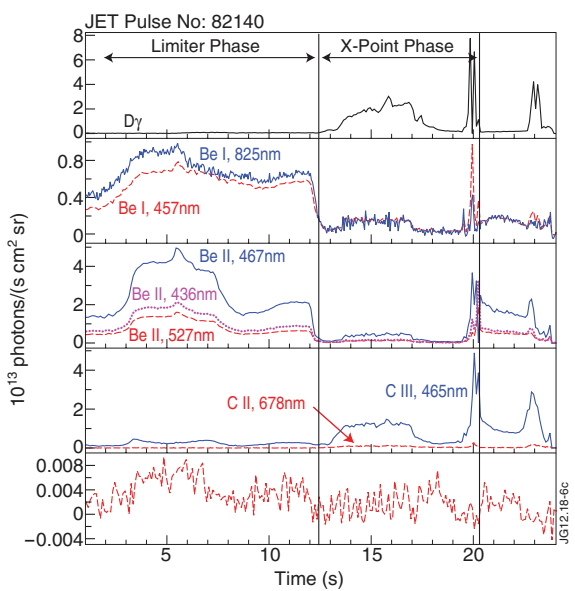

FIG. 6. Time traces of line intensities from selected impurities during a JET $\mathrm{H}$-mode discharge, measured by COS systems viewing the inner wall. The transition from limiter to $\mathrm{X}$-point phase is accompanied by a drop in $\mathrm{Be}$ emission and an increase in DI and C emission. The WI emission from the recessed NB shine through tiles is always very weak.

\section{ABSOLUTE INTENSITY CALIBRATION}

All radial LOS coupled to COS have been absolutely calibrated in situ by means of a radiometric light source placed inside the JET vessel in front of the whole optical path and operated by remote handling. ${ }^{4}$ A separate "ex-vessel" calibration was also performed, with the calibration lamp in front of the optical head and separate measurement of the window transmission, employing a green and a red laser and a retroreflector positioned by remote handling behind the window inside the JET vessel. The vertical LOS was only calibrated by means of ex-vessel calibration and separate window transmission measurement with the two colour-laser, since it was added to the KS8 diagnostic at a later stage, after the in situ calibrations were performed. The LOS coupled to the EOS could not be absolutely calibrated during the 2011 shutdown for technical reasons and will be back calibrated during the 2012 shutdown, when access to the optical head in the JET torus hall will be regained.

\section{RESULTS}

The KS8 diagnostic has been operating successfully since the first plasma pulse of the JET experimental campaigns with the ILW, which started in August 2011. It operates routinely in every JET discharge, monitoring photon fluxes from intrinsic impurities, such as $\mathrm{Be}, \mathrm{C}$, and $\mathrm{W}$ and injected gases $\left(\mathrm{N}\right.$ and $\mathrm{Ne}$ ), molecules (e.g., $\left.\mathrm{BeD}, \mathrm{D}_{2}, \mathrm{ND}\right)$ and main chamber and divertor recycling (typically $\mathrm{D} \alpha, \mathrm{D} \beta$, and $\mathrm{D} \gamma$ ). Examples of COS spectra from JET discharges are shown in Figure 4. An example of EOS spectrum during the limiter phase of a JET discharge is shown in Figure 5. Time traces of photon fluxes of selected impurity lines measured by COS systems along a LOS to the inner wall during a JET discharge are shown in Figure 6.

\section{CONCLUSIONS}

As part of the diagnostic enhancements accompanying the study of the Be/W wall, a new survey, visible spectroscopy diagnostic has been installed and commissioned in JET, employing a series of compact overview spectrometers with medium spectral resolution and a high resolution Echelle overview spectrometer. For each plasma discharge the diagnostic allows monitoring of intrinsic and injected impurities influxes (e.g., Be, C, N, W), molecular fluxes (BeD) and plasma recycling from the JET main chamber and outer divertor target.

\section{ACKNOWLEDGMENTS}

This work was supported by EURATOM and carried out within the framework of the European Fusion Development Agreement. The views and opinions expressed herein do not necessarily reflect those of the European Commission.

${ }^{1}$ G. F. Matthews et al., Phys. Scr. T145 014001 (2011).

${ }^{2}$ R. Neu, ASDEX Upgrade Team and EU PWI Taskforce, and JET EFDA Contributors, Plasma Phys. Controllled Fusion 53124040 (2011).

${ }^{3}$ S. Brezinsek, A. Pospieszczyk, G. Sergienko, P. Mertens, and U. Samm, Plasma Fusion Res., 3 S1041 (2008).

${ }^{4}$ T. M. Biewer, C. Belcher, I. Hassall, D. L. Hillis, G. Caveney, D. Scharpf, M. F. Stamp, C. Stunell, K.-D. Zastrow, and JET EFDA Contributors, Rev. Sci. Instrum. 83, 10D505 (2012). 